\title{
Magnesium Diboride: Better Late than Never
}

\begin{abstract}
With a superconducting transition temperature of $40 \mathrm{~K}$ and two superconducting gaps, $\mathrm{MgB}_{2}$ is full of surprises for both experimentalists and theorists.
\end{abstract}

\section{Paul C. Canfield and George W. Crabtree}

$T_{t}^{\mathrm{h}}$ he heyday of research into the basic properties of intermetallic superconductors took place between 1950 and 1980. During those years, the number of known superconducting intermetallic compounds (consisting of several metallic and metalloid elements) grew explosively, and superconducting transition temperatures $T_{\mathrm{c}}$ were pushed to just over $23 \mathrm{~K}\left(\mathrm{Nb}_{3} \mathrm{Ge}\right)$. (In comparison, the first superconductor, discovered by Heike Kamerlingh Onnes in 1911, was mercury, with $T_{\mathrm{c}}=4.15 \mathrm{~K}$ ). Research groups all over the world searched for higher and higher $T_{\mathrm{c}}$ values. The researchers were motivated by a basic desire to find an intrinsic limiting temperature for this intriguing quantum phase and by a very applied interest in making useful superconducting devices.

By the $1970 \mathrm{~s}$, an empirical glass ceiling seemed to have been hit. Transition temperatures were stuck at 23 $\mathrm{K}$, and some theorists proclaimed that higher transition temperatures were not possible. The basic research community became more interested in other issues in superconductivity, such as the interaction of local magnetic moments with superconductivity, heavy-fermion superconductors, organic superconductors, and, more recently, the high-temperature, copper oxide-based ceramic superconductors. With the discovery of high- $T_{\mathrm{c}}$ superconductivity over a decade ago, the $23-\mathrm{K}$ ceiling for $T_{\mathrm{c}}$ was shattered, giving us not only new horizons in transition temperature (the current record is about $160 \mathrm{~K}$ under pressure) but also a profusion of interesting phenomena like $d$-wave pairing symmetry, pseudogaps, stripes, and exotic pairing mechanisms. Conventional wisdom holds that these new horizons are enabled by the complexity of multielement compounds. These complex materials, in turn, have required complex processing to achieve the industrial production of superconducting wires and devices that is now taking place.

The January 2001 discovery that magnesium diboride becomes superconducting at about $40 \mathrm{~K}$ produced an explosion of enthusiasm and excitement. Although $40 \mathrm{~K}$ is indeed much cooler than $160 \mathrm{~K}$, it represents a near doubling of the previous record intermetallic $T_{\mathrm{c}}$ and means that $\mathrm{MgB}_{2}$ can be cooled to an operational temperature by either liquid hydrogen or readily available, fairly inexpensive, closed-cycle refrigerators. In addition, $\mathrm{MgB}_{2}$ is a

Paul Canfield is a senior physicist at Ames Laboratory and a professor in the department of physics and astronomy at lowa State University in Ames, Iowa. George Crabtree is a senior scientist and the director of the materials science division at Argonne National Laboratory in Argonne, Illinois. simple compound of two abundant, inexpensive elements. The long soughtafter, high-temperature, intermetallic superconductor had finally made its appearance-better late than never.

Although the initial interest in $\mathrm{MgB}_{2}$ arose solely from its high $T_{\mathrm{c}}$, further work revealed that $\mathrm{MgB}_{2}$ breaks new ground for superconductivity based on the well-known electron-phonon interaction. The material displays a plethora of remarkable features. $\mathrm{MgB}_{2}$ not only has important technological potential (figure 1 and the cover of this issue show images of $\mathrm{MgB}_{2}$ wire), but also will have a lasting impact on how the research community looks at and looks for superconductors.

The understanding of this material has grown at a whirlwind rate, driven by the immediacy of preprint postings on the Internet. The daily appearance of new research results brought theory and experiment into resonance and dramatically accelerated progress in the international community. Thanks to that positive feedback, the basic physical properties of $\mathrm{MgB}_{2}$ were delineated within a year and a half of its discovery. ${ }^{1}$

\section{Background and foreshadowing}

For decades after the discovery of superconductivity, the experimental data puzzled theorists. One of the key experiments that pointed toward the correct approach to understanding the phenomenon was the isotope effect, which showed that the transition temperature depends on the mass of the superconducting atoms. Motivated in part by those experiments, theorists recognized that lattice vibrations (phonons) could produce an attractive interaction between the like-charged electrons. The 1957 theory of John Bardeen, Leon Cooper, and J. Robert Schrieffer (BCS) explained superconducting properties quantitatively using an elegant microscopic theory and the electron-phonon interaction as the mechanism for pairing electrons.

At the most basic level, the superconducting ground state is a coherent superposition of Cooper pairs-pairs of electrons coupled by an attractive force. The attraction can arise from phonons in the following way. As a negatively charged electron moves through a lattice of positive ions, it attracts and locally distorts the lattice in its neighborhood. The residual distortion left in its wake produces a net positive charge that attracts a second electron.

The key feature of the superconducting state is the energy gap in the excitation spectrum. Below $T_{\mathrm{c}}$, this gap prevents scattering of the electrons and produces zero electrical resistance. The gap also manifests itself in tunneling experiments (in a manner similar to a semiconducting gap) that map its energy dependence and in thermodynamic experiments, such as specific heat measurements, that probe thermal excitations across the gap.

One of the most significant and influential predictions of the BCS theory is the description of $T_{\mathrm{c}}$ in terms of two fundamental constants (Boltzmann's constant $k_{\mathrm{B}}$ and 


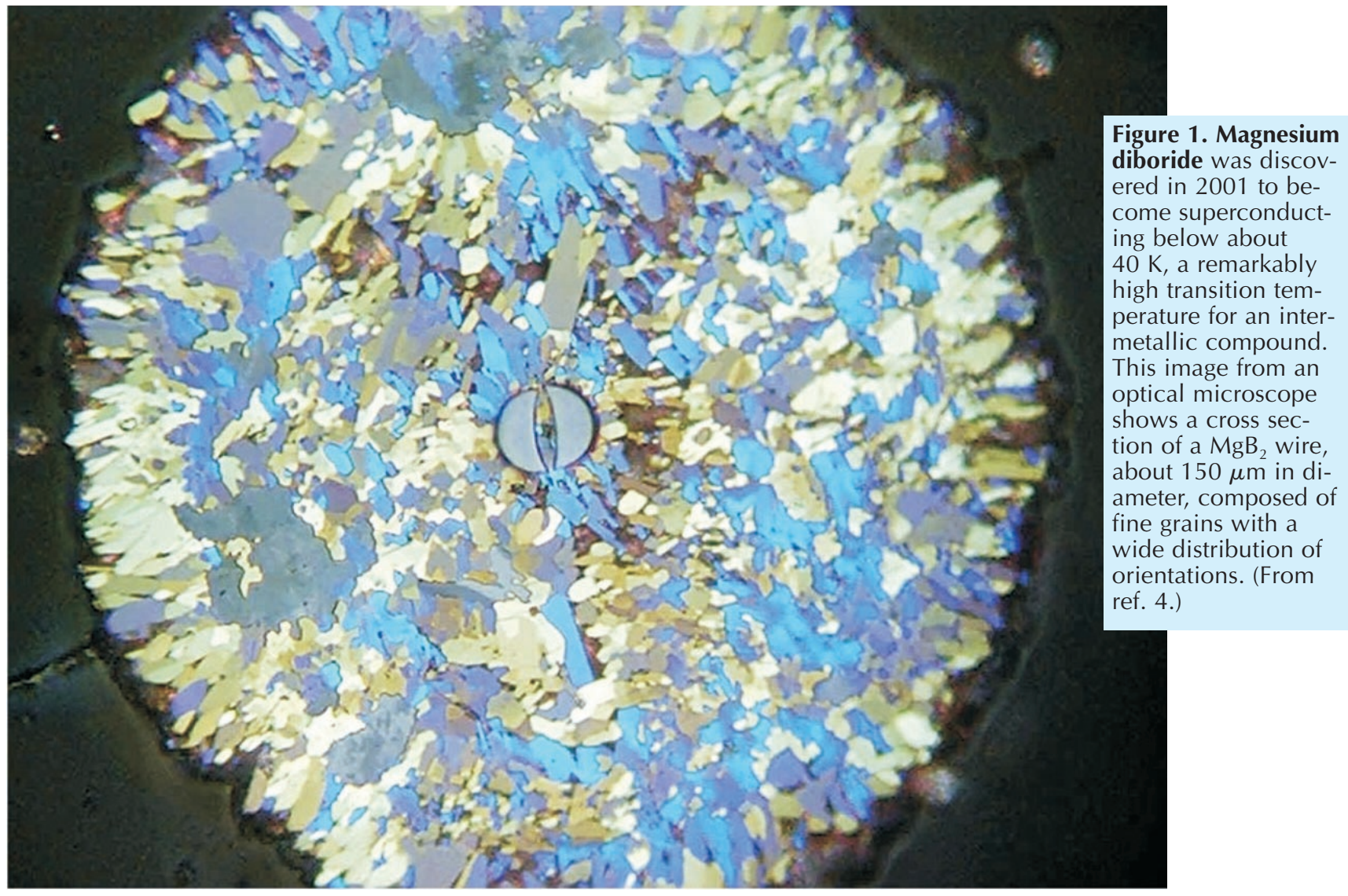

Planck's constant $\hbar$ ) and three basic materials parameters:

$$
k_{\mathrm{B}} T_{\mathrm{c}}=1.13 \hbar \omega_{\mathrm{D}} \exp \left[-1 / V N\left(E_{\mathrm{F}}\right)\right] .
$$

The Debye frequency $\omega_{\mathrm{D}}$ is the characteristic frequency of the acoustic lattice vibrations. It sets the energy scale of the attractive interaction between Cooper pairs. In the simplest model of a solid, the atoms can be viewed as masses $m$ connected by springs with spring constant $k$. From high-school physics (or what should be high-school physics), the characteristic frequency of such an idealized model is $\omega=(k / m)^{1 / 2}$. This simple observation, combined with the BCS formula above, is the essence of the isotope effect. Within this framework, $T_{\mathrm{c}}$ is inversely proportional to the square root of the masses of the constituent elements. So, to first order, compounds with lighter elements have a better shot at higher values of $T_{\mathrm{c}}$.

The electron-electron attractive interaction $V$ is a caliper of the strength of the electron-phonon interaction. If the electron-phonon interaction is increased, $V$ and $T_{\mathrm{c}}$ rise, but if it becomes too large, it can induce a phase transition to a different structure, often with vastly inferior superconducting properties. For that reason, researchers have often sought high values of $T_{\mathrm{c}}$ near, but just short of, structural phase transitions; that approach is the most pragmatic method of maximizing $V$ for a given structure.

The density of itinerant electron states $N\left(E_{\mathrm{F}}\right)$, where $E_{\mathrm{F}}$ is the Fermi energy, provides a caliper of the number of electrons that can participate in the superconducting state. The larger $N\left(E_{\mathrm{F}}\right)$, the larger $T_{\mathrm{c}}$. Given that transition metals with partially occupied $d$ shells tend to have large values of $N\left(E_{\mathrm{F}}\right)$, conventional wisdom held that high values of $T_{\mathrm{c}}$ could be found in transition-metal compounds.

Experimentally, it is possible to tune $N\left(E_{\mathrm{F}}\right)$ by varying the chemical composition in an isostructural series of compounds. Using light elements can raise $\omega_{\mathrm{D}}$. Experimental searches for new superconductors often placed a much larger emphasis on $N\left(E_{\mathrm{F}}\right)$ and $\omega_{\mathrm{D}}$ than on $V$, because the electron-phonon coupling-and hence $V$-is hard to predict or control.

\section{$\mathrm{MgB}_{2}$ : Discovery and isotope effect}

Based on these experiments, theories, and prejudices, groups around the world searched for ternary or quaternary compounds that were rich in light elements such as lithium, boron, carbon, and magnesium. Jun Akimitsu's group at Aoyama Gakuin University in Japan explored the titanium-boron-magnesium ternary phase diagram. That system has two light elements as well as the transition metal Ti, which could provide $3 d$ electrons to boost $N\left(E_{\mathrm{F}}\right)$. Two years ago, ${ }^{2}$ the researchers indeed found a remarkably high $T_{\mathrm{c}}$, but in a simple binary compound: $\mathrm{MgB}_{2}$ entered the superconducting state just below $40 \mathrm{~K}$ (see figure 2). In early January 2001, Akimitsu reported their results at a meeting in Sendai, Japan. His announcement set off a rush of experimental and theoretical work to confirm and explore that remarkably high transition temperature in a binary intermetallic compound (see PHYSICS TODAY, April 2001, page 17).

Whereas $\mathrm{MgB}_{2}$ is a simple binary compound (only three atoms per hexagonal unit cell), it is hard to make by conventional methods. For starters, elemental $\mathrm{Mg}$ has a high vapor pressure. Furthermore, $\mathrm{MgB}_{2}$ decomposes rather than melts and does not have any accessible liquid-solid transitions at ambient pressures. ${ }^{1,3}$ Simple crystal growth of $\mathrm{MgB}_{2}$ thus appears to be intractable. (This difficulty is one of the reasons that superconductivity in $\mathrm{MgB}_{2}$ was not discovered 40 years ago.) Fortunately, $\mathrm{MgB}_{2}$ can be synthesized by a simple, alternate route: reaction of $\mathrm{B}$ (in any number of forms) with $\mathrm{Mg}$ vapor, generally at a temperature of about $900^{\circ} \mathrm{C}$ for as little as a couple of hours. To date, this 
Figure 2. Magnesium diboride's superconducting transition below about $40 \mathrm{~K}$ is clearly seen in the temperature dependence of the electrical resistivity $\rho$. The insets show the effect of replacing boron-10 with boron-11: a reduction of about $1 \mathrm{~K}$ in the transition temperature. The isotope effect is seen in transport properties, such as normalized temperature-dependent resistivity in the top left plot, and in bulk thermodynamic properties, such as the normalized magnetization $M$ plotted in the lower right.

method has been used to form $\mathrm{MgB}_{2}$ powder, sintered pellets, wires, and films. ${ }^{1,3-5}$ The wires shown in figure 1 and on the cover of this issue were made from $B$ filaments using this technique. More recently, highpressure, high-temperature methods of growing $\mathrm{MgB}_{2}$ have been developed, and single crystals with masses approaching a milligram are becoming available., ${ }^{1,6}$

By exposing isotopically pure ${ }^{11} \mathrm{~B}$ or ${ }^{10} \mathrm{~B}$ powder to $\mathrm{Mg}$ vapor, sintered pellets of $\mathrm{Mg}^{11} \mathrm{~B}_{2}$ and $\mathrm{Mg}^{10} \mathrm{~B}_{2}$ are simple to synthesize. The first measurements ${ }^{1,3}$ of the isotope effect revealed the importance of the electron-phonon interaction for the Cooper pairing mechanism in $\mathrm{MgB}_{2}: T_{\mathrm{c}}$ shifted by $1.0 \mathrm{~K}$ when ${ }^{11} \mathrm{~B}$ was substituted for ${ }^{10} \mathrm{~B}$, as shown in the figure 2 insets. The isotope shift associated with $\mathrm{Mg}$ can be measured, too. Surprisingly, substitution of ${ }^{24} \mathrm{Mg}$ for ${ }^{25} \mathrm{Mg}$ produced almost no shift in $T_{\mathrm{c}}{ }^{1,7}$ The contrast of these two results is striking and immediately indicates a highly selective electron-phonon interaction. A more subtle experimental effect was also found: The total isotope shift for $\mathrm{Mg}$ plus B is significantly smaller than that predicted by the BCS theory. ${ }^{1,7}$ The origin of the discrepancy cannot be inferred from the experiment. It turns out to be due to another unique feature of $\mathrm{MgB}_{2}$ : the nature and extreme strength of the electron-phonon coupling responsible for superconductivity in this compound. ${ }^{1,8}$

If $\mathrm{MgB}_{2}$ is an electron-phonon superconductor, how can it have such a high $T_{\mathrm{c}}$ ? From the BCS formula, $T_{\mathrm{c}}$ depends on only three materials parameters: the characteristic phonon energy $\hbar \omega_{\mathrm{D}}$, the electronic density of states $N\left(E_{\mathrm{F}}\right)$, and the electron-phonon interaction giving rise to $V$. The phonon energies of $\mathrm{MgB}_{2}$ are indeed rather high, but not especially different from other diborides and light-element binary compounds that have much lower values of $T$. The density of states is low- $\mathrm{MgB}_{2}$ has no $d$ electrons. The BCS formula leaves only one other source for high $T_{\mathrm{c}}$ : the electron-phonon coupling as expressed through $V$. This simple analysis turns out to be prescient: The selective coupling between specific electronic states and specific phonons is the key feature that drives superconductivity and other interesting properties of this compound.

\section{All bands are not created equal}

Magnesium diboride has a simple crystal structure consisting of hexagonal $\mathrm{Mg}$ layers alternating with honeycomb B layers, as shown in figure $3 \mathrm{a}$. The $\mathrm{Mg}$ ions donate electrons to the conduction bands, but the $\mathrm{Mg}$ atomic orbitals play only a small role in the conduction process-it's the honeycomb planes of $\mathrm{B}$ that determine the electronic properties. ${ }^{1,8}$
The B electronic states can be best understood by recalling the benzene molecule from high-school chemistry (or what should be high-school chemistry). In benzene, the $s p^{2}$ carbon orbitals overlap, creating $\sigma$ bonds between neighboring atoms in the plane of the molecule. The remaining carbon $p$ orbitals extend above and below the plane and create the $\pi$ bonds. The electrons in both bonds are delocalized among the six $\mathrm{C}$ atoms in the benzene ring, although they may not jump from the $\sigma$ to the $\pi$ bonds. In $\mathrm{MgB}_{2}$, the $\mathrm{B}$ honeycomb plays the role of the $\mathrm{C}$ ring in benzene, with the key differences that the rings form an extended two-dimensional network and electrons are delocalized throughout the honeycomb. The $\sigma$ and $\pi$ bonds in benzene become $\sigma$ and $\pi$ bands in $\mathrm{MgB}_{2}$, with very little electron hopping between them. In $\mathrm{MgB}_{2}$, the $\pi$ bands connect adjacent B layers (through the inert $\mathrm{Mg}$ ions), which allows metallic conduction perpendicular to the B sheets (conventionally called the $c$-direction) as well as parallel to the B sheets. The $\sigma$ electrons are restricted to the B layer and conduct only in this basal plane. The geometry of the electron densities associated with these bands is depicted in figure $3 \mathrm{~b}$. The band structure can be represented compactly in momentum space by its Fermi surface, the energy contour separating filled from empty electronic states, shown in figure 3c.

These two nearly noninteracting bands of different dimensionality are key ingredients in $\mathrm{MgB}_{2}$. The other key ingredient is the bands' special sensitivity to phonons. In conventional superconductors, the electron-phonon interaction creates Cooper pairs of approximately equal pairing strength, distributed evenly over the Fermi surface. In $\mathrm{MgB}_{2}$, there is one high-energy (about $570 \mathrm{meV}$ ) optical phonon (denoted $E_{2 g}$ for its symmetry), associated with the in-plane motion of the B atoms, that couples very strongly to electrons in the 2D $\sigma$ band. The strong coupling of the $\mathrm{B}$ motion to the $\sigma$ states originates in the special covalent nature of the $\sigma$ band. Unlike conventional metallic bands, the $\sigma$ band has charge concentrated along the B-B axes (see figure $3 \mathrm{~b}$ ) rather than spread throughout the unit cell. Thus, when the B atoms move in the plane, the charge must redistribute significantly to accommodate the 

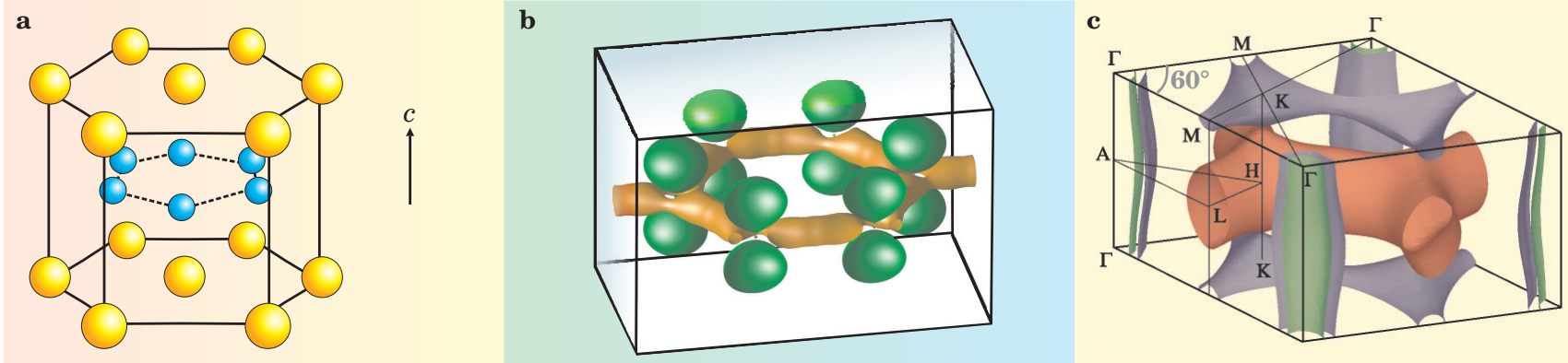

Figure 3. Structure of magnesium diboride. (a) The crystal structure of $\mathrm{MgB}_{2}$ features hexagonal planes of magnesium (yellow) interleaved with honeycomb layers of boron (blue). (b) The boron atoms form a two-dimensional network of $\sigma$ bonds and a three-dimensional network of $\pi$ bonds; the two networks yield corresponding bands of electronic states. Shown here is a contour of constant charge density. The golden hexagonal network is associated with the $\sigma$ bands, whereas the six pairs of green lobes above and below the boron plane are associated with the $\pi$ bands. The $\pi$-band charge density extends in both the parallel and perpendicular directions to form 3D conduction states. (Courtesy of O. Jepsen, adapted from I. I. Mazin et al., ref. 8.) (c) The Fermi surface of $\mathrm{MgB}_{2}$. The vertical sections of cylinders at the corners are associated with the $\sigma$ bands; the more 3D network of tunnels and caves in the center of the zone is associated with the $\pi$ bands. The letters designate the symmetry points of the hexagonal Brillouin zone in momentum space. (Adapted from J. Kortus et al., ref. 8.)

change, and the golden hexagonal network of charge in figure $3 \mathrm{~b}$ becomes substantially distorted. The distortion shifts the energy of the electronic states and accounts for the large coupling energy. This picture of two bands of different dimensionality with strong selective electronphonon coupling is confirmed by detailed measurements of the electronic structure, such as experiments that use quantum oscillations of the magnetization (the de Haas-van Alphen effect) to infer the local Fermi surface geometry and the electron density of states. ${ }^{1,9}$

The $E_{2 g}$ phonon has a large impact on the properties of $\mathrm{MgB}_{2}$ because it affects the conduction electrons; had it coupled to the fully filled or empty bands, the consequences would be far less dramatic. Physically, this dominant phonon mode sets the energy scale for the superconductivity and its characteristic energy replaces $\hbar \omega_{\mathrm{D}}$ as the prefactor in the BCS equation for $T_{\mathrm{c}}$ (roughly a factor of 10 enhancement!). In addition, the highly selective electron-phonon interaction gives rise to the substantial boron-and negligible magnesium-isotope shift. It also answers the question of how $T_{\mathrm{c}}$ is so high, given the relatively low $N\left(E_{\mathrm{F}}\right)$. $^{1,10}$

\section{Basic properties}

How do the special features of the band structure and elec-

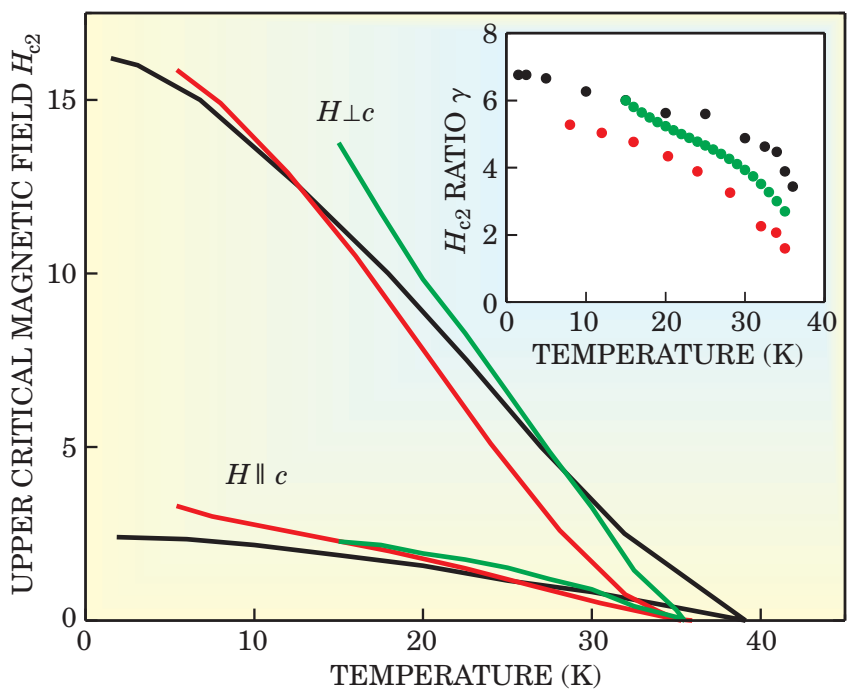

tron-phonon interaction in $\mathrm{MgB}_{2}$ manifest themselves? Almost every possible measurement shows their effect. Even the normal-state resistivity shown in figure 2 is curious in its own right., ${ }^{1,4}$ One of the old rules of thumb for intermetallic superconductors was that a high $T_{\mathrm{c}}$ is associated with large room-temperature resistivity arising from the strong electron-phonon scattering of electrons in the normal state. Magnesium diboride, though, has a low roomtemperature resistivity (about $10 \mu \Omega \mathrm{cm}$ ) and a very low resistivity just above $T_{\mathrm{c}}$ (around $0.5 \mu \Omega \mathrm{cm}$ ). Its resistivity is thus comparable to bulk copper wire. In contrast, the superconductor $\mathrm{Nb}_{3} \mathrm{Sn}$ has a resistivity of $80 \mu \Omega \mathrm{cm}$ at room temperature and $10 \mu \Omega \mathrm{cm}$ just above its $T_{\mathrm{c}}$. The low resistivity of $\mathrm{MgB}_{2}$ is simply a consequence of having two bands with very different electron-phonon coupling strengths. In essence, the two bands take turns shorting each other out. (To understand such behavior qualitatively, look at figure $3 \mathrm{~b}$ and think of the contours as forming parallel networks of resistors.) The high-temperature resistivity is dominated by the $3 \mathrm{D} \pi$ band, which has the smaller electron-phonon coupling, whereas the superconductivity is primarily due to the larger coupling in the $2 \mathrm{D}$ $\sigma$ band. This simple picture implies a moderate anisotropy in the normal-state resistivity, with a larger resistivity in the $c$-direction than in the basal plane; such anisotropy has indeed been observed. ${ }^{1}$

Another startling aspect of $\mathrm{MgB}_{2}$ is the anisotropy of its upper critical magnetic field $H_{c 2}$. The upper critical field is the highest magnetic field (at a given temperature) for which a material will remain a bulk superconductor. The magnetic field strength needed to destroy superconductivity in bulk $\mathrm{MgB}_{2}$ depends on the orientation of the field with respect to the crystal axes, as shown in figure 4 .

Figure 4. Upper critical magnetic field $\boldsymbol{H}_{\mathrm{c} 2}$ for magnesium diboride is anisotropic, reflecting the underlying anisotropic electronic bands. $H_{\mathrm{c} 2}$ is the magnetic field sufficient to destroy superconductivity in bulk samples. The data shown here were inferred from measurements on polycrystalline ${ }^{11}$ (red) and single crystal ${ }^{12}$ (black and green) samples. The lower curves are $H_{c 2}^{\| c}$ for the magnetic field applied along the $c$-axis (perpendicular to the basal hexagonal planes in figure $3 a)$, and the upper curves are $H_{c 2}^{\perp c}$ for the magnetic field applied within the basal plane. The inset shows the anisotropy ratio $\gamma \equiv H_{\mathrm{c} 2}^{\perp c} / H_{\mathrm{c} 2}^{\| c}$ from each of these data sets. 
Figure 5. The electronic contribution to the specific heat $C_{\mathrm{p}}$ presented the first indication of the presence of two superconducting gaps in $\mathrm{MgB}_{2}$. The data plotted here were taken on a polycrystalline sample of $\mathrm{MgB}_{2}$ and have been adjusted to remove the lattice contribution. ${ }^{13}$ The red curve shows the prediction from the Bardeen-CooperSchrieffer theory for a single superconducting gap in zero magnetic field. The shoulder at low temperatures and fields indicates the existence of a second gap. (Adapted from F. Bouquet et al., ref 13.)

Despite being taken from samples of differing form (polycrystalline $\mathrm{e}^{1,11}$ and single-crystal $\left.{ }^{1,12}\right)$ and quality $(36 \mathrm{~K}<$ $\left.T_{\mathrm{c}}<40 \mathrm{~K}\right)$, the $H_{\mathrm{c} 2}$ data show good agreement. The anisotropy, quantified as the ratio $\gamma \equiv$ $H_{\mathrm{c} 2}^{\perp c} / H_{\mathrm{c} 2}^{\| c}$, not only is large, but it has an unusual temperature dependence (see figure 4 inset). At low temperatures, $\gamma$ is about 6 , a value close to that expected theoretically from the geometry of the Fermi surface associated with the $\sigma$ band (figure 3c). ${ }^{1,11}$ This observation strongly suggests that the low-temperature, high-field superconducting state is dominated by just the $\sigma$ band.

Specific heat measurements of $\mathrm{MgB}_{2}$ below $T_{\text {c }}$ gave the first indication that the $\sigma$ and $\pi$ bands of $\mathrm{MgB}_{2}$ may have separate and distinct superconducting gaps. ${ }^{1,13}$ Figure 5 shows the electronic specific heat of $\mathrm{MgB}_{2}$ in zero and high applied magnetic field. The temperature dependence of the electronic contribution to the specific heat deviates markedly from that predicted by BCS theory. The most important deviation is the low-temperature shoulder, associated with a second, smaller gap, that is very rapidly suppressed by an applied field. The nature and implications of these two gaps have been the focus of much of the recent basic research on $\mathrm{MgB}_{2}$.

\section{Two superconducting gaps}

The strong electron-phonon coupling in the 2D $\sigma$ bands and weak coupling in the $3 \mathrm{D} \pi$ bands lead to a striking qualitative feature of superconductivity in $\mathrm{MgB}_{2}$ : the simultaneous existence of two energy gaps in the same material.,14 The concept of two gaps in a single superconductor has been considered before ${ }^{15}$ but $\mathrm{MgB}_{2}$ is the first example of a two-gap superconductor in which the effects are so dramatically expressed. ${ }^{1,10}$

The two gaps in $\mathrm{MgB}_{2}$ arise naturally from the different strengths of the electron-phonon coupling in the $\sigma$ and $\pi$ bands. If the two sets of electrons were independent, the physics would be straightforward: Two noninteracting superconducting condensates share the same crystalline framework but have different transition temperatures and distinct superconducting properties. The two sets of electrons do interact, though, albeit weakly, through scattering from states in one band to states in the other and through Coulomb repulsion. This small interaction provides the richness and subtlety that permeates all the properties of $\mathrm{MgB}_{2}$. For example, a small interaction causes both bands to become superconducting at the same temperature; a large interaction, in contrast, would mix the two bands thoroughly and wash out the distinction in their energy gaps and dimensionality. In $\mathrm{MgB}_{2}$, the two bands and the two very differently sized superconducting gaps are quite distinct and manifest themselves clearly.
The two gaps can be seen most directly in the superconducting tunneling behavior. Figure 6 a shows the topographic image, taken with a scanning tunneling microscope, of a polycrystalline sample of $\mathrm{MgB}_{2}$ with four grains of different crystallographic orientation. By fixing the STM above the sample and measuring the differential conductance as a function of applied voltage, the tunneling spectrum of the sample beneath the STM tip can be obtained. Such spectra were taken on a dense grid of points in the indicated box straddling grains 1 and 2 . The spectra showed a pronounced change exactly at the grain boundary: the spectra from grain 1 show dominant lowvoltage conductance peaks, whereas those from grain 2 feature dominant high-voltage peaks. In qualitative terms, the positions of the peaks measure the sizes of the superconducting energy gaps in the $\mathrm{MgB}_{2}$ sample. The remarkable feature of these spectra is their strong sensitivity to the change in grain orientation relative to the tunneling direction. The spectrum in grain 1 is typical of tunneling along the $c$-direction: It probes predominantly the $\pi$ band gap, since the $\sigma$ band conducts principally in the basal plane and cannot couple to tunneling currents along the $c$ direction. The spectrum in grain 2 is typical of tunneling currents in the basal plane, which couple to both the $\sigma$ and $\pi$ bands. The temperature dependence of the two gaps can be tracked, as shown in figure $6 \mathrm{~b}$, using the directional sensitivity of the tunneling spectra to identify the gaps. ${ }^{1,16}$

The tunneling data explain the anomalous specific heat data in figure 5 . The behavior near $T_{\mathrm{c}}$ is determined by the large $2 \mathrm{D}$ gap, which produces a step as expected for a conventional one-gap superconductor. The size of the step is smaller than expected, however, because the smaller 3D gap of the $\pi$ bands easily admits thermal excitations. At lower temperatures, a large shoulder appears in the specific heat as the smaller 3D gap cuts off lowenergy thermal excitations. ${ }^{1,13}$

The simultaneous presence of two superconducting condensates with different gaps and dimensionalities leads to a host of potentially new phenomena. Each condensate has its own set of natural characteristic lengths reflecting its own gap size, electron density, and anisotropy. Unraveling the physical behavior of the interacting condensates becomes a fascinating game of hide-and-seek: Which characteristic length controls which property? For example, consider once more the anisotropic $H_{\mathrm{c} 2}$ data shown in figure 4 . Near $T_{\mathrm{c}}$ in low magnetic field, both condensates contribute to the upper critical field, and defining a single char- 

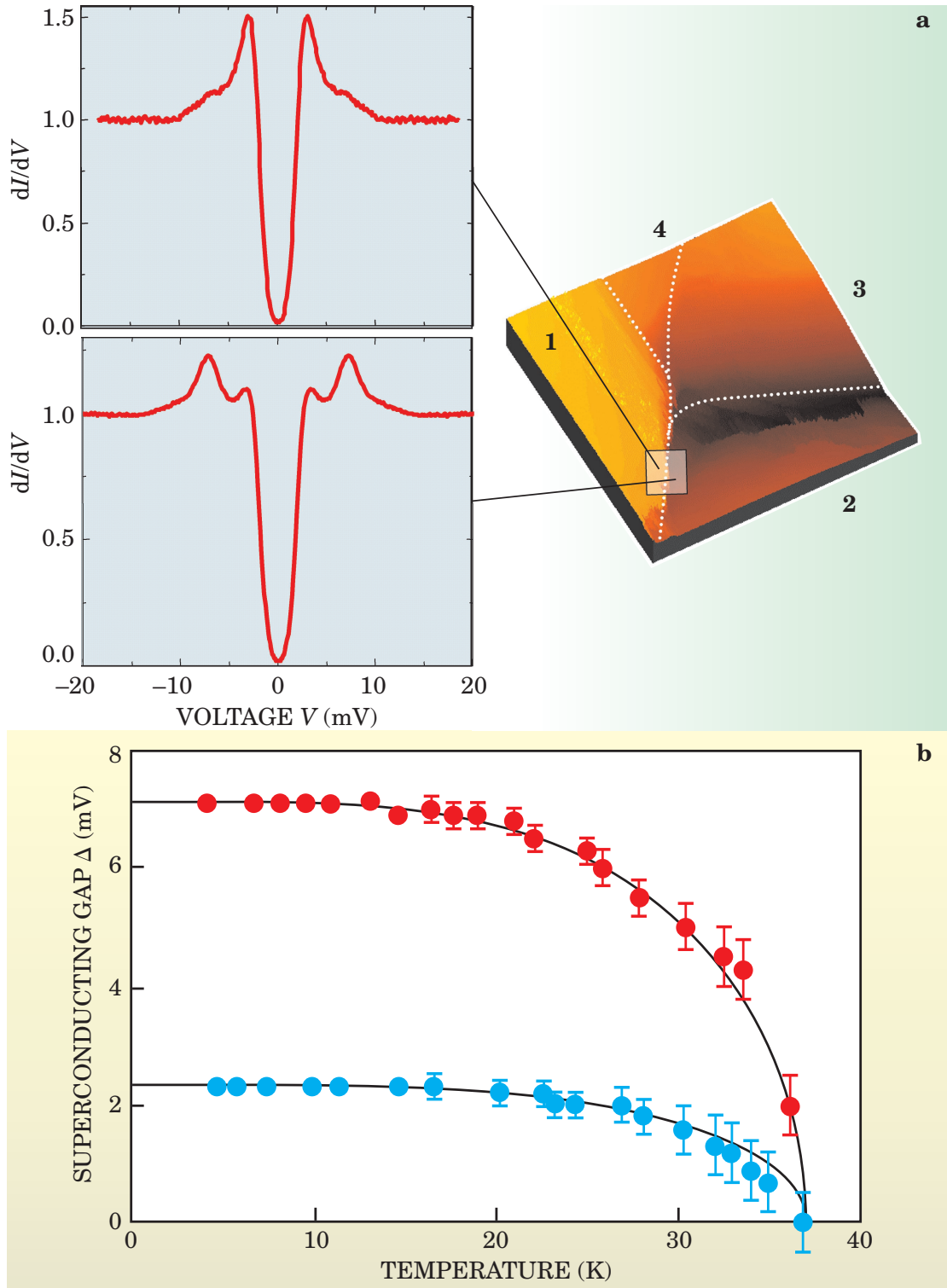

Figure 6. Tunneling experiments clearly show the presence of two superconducting gaps in magnesium diboride. (a) This topographic image (right) taken by a scanning tunneling microscope shows a section of a polycrystalline sample, $150 \mathrm{~nm}$ on a side, containing four grains. Tunneling spectra (left) measured with an STM change dramatically at the boundary between grains 1 and 2 . The spectra are plotted as the differential conductance $\mathrm{d} I / \mathrm{d} V$ (where $I$ is the tunneling current), normalized to the high-voltage conductance. Peaks in the spectra identify the superconducting gaps. (b) The sensitivity of the spectra to the tunneling direction allows the two gaps to be distinguished and followed as a function of temperature. The red points are the large gap of the two-dimensional $\sigma$ band; the blue are the small gap of the threedimensional $\pi$ band. (Adapted from M. lavarone et al., ref. 16.)

sented (in tabular form) in $1957 .{ }^{17}$ The detection of the superconducting transition was barely missed. Finally, the theory for a superconductor with two distinct gaps was proposed in $1959,{ }^{15}$ but lay fallow for lack of a concrete example. One has to wonder how the world may have been different if $40-\mathrm{K}$ superconductivity in $\mathrm{MgB}_{2}$ had been discovered in 1960 , before $\mathrm{Nb}_{3} \mathrm{Sn}$ and $\mathrm{Nb}-\mathrm{Ti}$ alloys were developed as practical superconductors. Would we now be generating magnetic fields, taking magnetic resonance imaging scans, measuring tiny magnetic moments, and relaying cell-phone calls using technology based on $\mathrm{MgB}_{2}$ ?

As it is, $\mathrm{MgB}_{2}$ has opened our eyes to previously unrealized possibilities for electron-phonon superconductivity. Higher transition temperatures and other compounds now occupy our dreams, and novel basic phenomena

acteristic length in terms of a single energy gap is problematic.,16 At higher field and lower temperature, the smaller 3D gap is suppressed and the upper critical field is dominated increasingly by the stronger $2 \mathrm{D}$ gap. Measurements of the anisotropic temperature-dependent upper critical field reflect this behavior. They show an anomalous upward curvature in the critical field in the basal plane, and a corresponding increase by more than a factor of two in the anisotropy ratio $\gamma$ as the temperature is lowered from $T_{\text {c }}$ to $5 \mathrm{~K}$ (see figure 4 inset). ${ }^{1,11,12}$

\section{Past, present, and future}

It is ironic that the remarkable superconductivity in $\mathrm{MgB}_{2}$ had to wait until the 21st century to be discovered. All of the conditions were ripe more than 40 years ago: Electron-phonon pairing monopolized superconductivity research, binary superconductors like $\mathrm{Nb}_{3} \mathrm{Sn}$ and $\mathrm{Nb}_{3} \mathrm{Ge}$ were already well known, and vigorous searches for new intermetallic superconductors were topical. Magnesium diboride was not even a new compound in those days; it had been known since the early 1950s. Its specific heat was measured down through $40 \mathrm{~K}$ and the rather sparse data were pre- such as two-gap superconductivity drive theory and experiment in new directions. Unlike the still-controversial cuprates, the physical picture and quantitative theoretical description for $\mathrm{MgB}_{2}$ are now available. We understand the conditions needed for high transition temperatures and for coexistence of two gaps of very different sizes. Theorists have begun to propose hypothetical compounds that may satisfy these conditions, but Nature often frustrates such designs by refusing to let these compounds form. Nevertheless, we think we know what we're looking for. Bandstructure and electron-phonon calculations for designer compounds are becoming accessible and may be incisive.

Magnesium diboride opens new avenues for basic and applied research. On the basic side, can we find other materials with multiple gaps and high transition temperatures? The interplay of structure, strongly selective electron-phonon coupling, and weakly interacting bands of different character may well hold still more subtle secrets. Magnesium diboride itself presents an intellectual challenge: to understand the consequences of two-gap superconductivity in all its glory. And although we have not discussed the possible applications of $\mathrm{MgB}_{2}$ in this article, $\mathrm{MgB}_{2}$ does appear to be a very promising material. For pure, 
Next Generation AFM Technology Today

\section{The New MFP-3D'}

Now shipping with the first small, low noise cantilever- the Bio-Lever

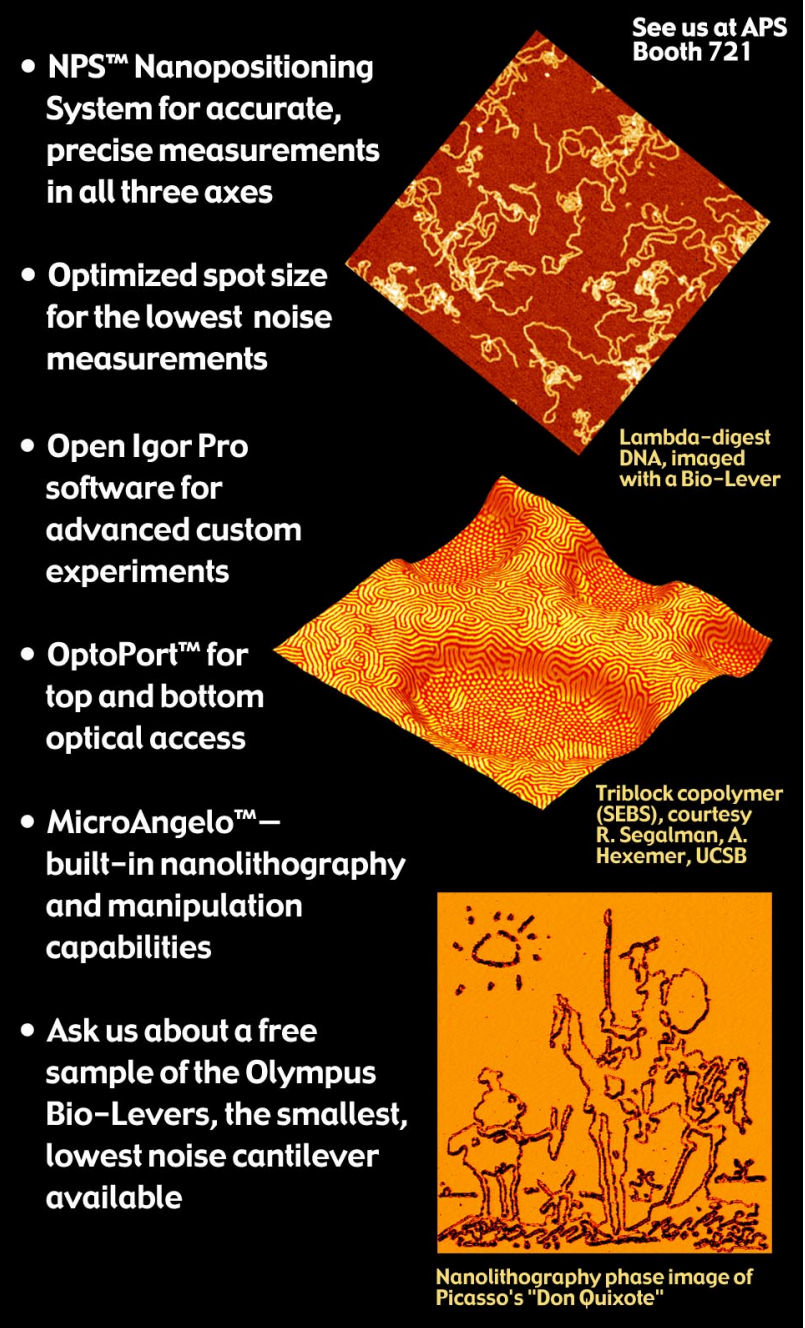

The MFP-3D's advanced capabilities and expandability make it an ideal system today and for the future. Call us for a demo or email us at sales@AsylumResearch.com.

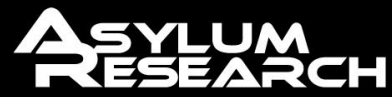
www.AsylumResearch.com 805-685-7077

APS Show-Booth \#721

Circle number 19 on Reader Service Card polycrystalline samples, the critical current density-a benchmark of a superconductor's suitability for many areas of use-approaches $10^{6} \mathrm{~A} / \mathrm{cm}^{2}$ at low fields and temperatures, and recent work indicates that this value can be increased by about an order of magnitude by the judicious addition of impurities. ${ }^{1,4,18}$ In a similar manner, the upper critical field has been almost tripled. ${ }^{1,18}$ The values of the critical current density and upper critical field start to exceed those found for the industrial standards $\mathrm{Nb}_{3} \mathrm{Sn}$ and $\mathrm{Nb}-\mathrm{Ti}$. The quality of $\mathrm{MgB}_{2}$ thin films continues to improve, and there is hope that devices such as superconducting quantum interference devices (SQUIDs) and filters may not be too difficult to make. And given that the mass density of $\mathrm{MgB}_{2}$ is low, as is the electrical resistivity of the normal state, magnets and cables made of $\mathrm{MgB}_{2}$ may be significantly lighter and cheaper than those of $\mathrm{Nb}_{3} \mathrm{Sn}$ and $\mathrm{Nb}-\mathrm{Ti}$. Such potential looks very promising, especially when combined with a readily accessible $T_{c}$ of about $40 \mathrm{~K}$.

Finally, we note that $\mathrm{MgB}_{2}$ is a poster child for an important observation about condensed matter physics. Superconductivity in this simple binary compound lay undiscovered until the new millennium, despite extensive searches over the past 80 years. Although we now understand the basic picture of superconductivity in $\mathrm{MgB}_{2}$, the behavior was not predicted a priori. The continued experimental search for new materials and new behavior in binary, ternary, quaternary, and other compounds is one of the most important engines driving progress in condensed matter physics. To quote Sophocles: "Look and you will find it-what is unsought will go undetected."

We thank Sergey Bud'ko, Maria Iavarone, and Goran Karapetrov for providing figures and useful discussions. We both gratefully acknowledge support from the US Department of Energy, Office of Science, Office of Basic Energy Sciences.

\section{References}

1. A comprehensive review of the first two years' research on magnesium diboride can be found in the March 2003 special issue of Physica $C, \mathbf{3 8 5}(1-2)$. That compilation of papers from numerous research groups working on $\mathrm{MgB}_{2}$ is a useful resource for many of the topics discussed in this article.

2. J. Nagamatsu et al., Nature 410, 63 (2001).

3. S. L. Bud'ko et al., Phys. Rev. Lett. 86, 1877 (2001).

4. P. C. Canfield et al., Phys. Rev. Lett. 86, 2523 (2001); R. A Ribeiro et al., Physica C 382, 194 (2002).

5. S. R. Shinde et al., Appl. Phys. Lett. 79, 227 (2001).

6. S. Lee et al., Physica C 378-381, 33 (2002); J. Karpinski et al., Supercond. Sci. Technol. 16, 221 (2003).

7. D. G. Hinks, H. Claus, J. D. Jorgensen, Nature 411, 457 (2001)

8. J. Kortus et al., Phys. Rev. Lett. 86, 4656 (2001); J. M. An, W. E. Pickett, Phys. Rev. Lett. 86, 4366 (2001); I. I. Mazin et al., http://arXiv.org/abs/cond-mat/0212417.

9. E. A. Yelland et al., Phys. Rev. Lett. 88, 217002 (2002).

10. H. J. Choi et al., Nature 418, 758 (2002).

11. S. L. Bud'ko, V. G. Kogan, P. C. Canfield, Phys. Rev. B 64 180506 (2001); S. L. Bud'ko, P. C. Canfield, Phys. Rev. B 65, 212501 (2002).

12. M. Angst et al., Phys. Rev. Lett. 88, 167004 (2002); U. Welp et al., Phys. Rev. B 67, 012505 (2003).

13. F. Bouquet et al., Phys. Rev. Lett. 87, 047001 (2001); Y. Wang, T. Plackowski, A. Junod, Physica C 355, 179 (2001).

14. A. Y. Liu, I. I. Mazin, J. Kortus, Phys. Rev. Lett. 87, 087005 (2001).

15. H. Suhl, B. T. Matthias, L. R. Walker, Phys. Rev. Lett. 3, 552 (1959); G. Binnig et al., Phys. Rev. Lett. 45, 1352 (1980).

16. P. Szabó et al., Phys. Rev. Lett. 87, 137005 (2001); H. Schmidt et al., Phys. Rev. Lett. 88, 127002 (2002); M. Iavarone et al., Phys. Rev. Lett. 89, 187002 (2002); M. R. Eskildsen et al., Phys. Rev. Lett. 89, 187003 (2002).

17. R. M. Swift, D. White, J. Am. Chem. Soc. 79, 3641 (1957).

18. D. K. Finnemore et al., Phys. Rev. Lett. 86, 2420 (2001); C. B. Eom et al., Nature 411, 558 (2001)

40 March 2003 Physics Today 